\title{
In Situ Measurement of Localized Surface Plasmon Resonance of Metal Nanoparticles in different surrounding
}

\author{
Pin Ann Lin ${ }^{1,2}$, Jonathan Winterstein ${ }^{3}$, Henri Lezec ${ }^{1}$, Renu Sharma ${ }^{1}$ \\ 1. Institute for Research in Electronics and Applied Physic, University of Maryland, College park, \\ USA \\ 2. Center for Nanoscale Science and Technology, National Institute of Standards and Technology, \\ Gaithersburg, MD, USA \\ ${ }^{3 .}$ FEI Co., Hillsboro, OR, USA
}

The high-field regions that occur at metal nanoparticle (NP) surfaces as a consequence of their localized surface plasmon resonances (LSPR), make them attractive for catalysis, optical, and waveguide applications as well as nanosensors. Optical spectroscopic methods, currently employed to measure the dependence of LSPR modes and intensity on metal nanoparticle size, shape and surrounding (support and gaseous environment), are limited to a spatial resolution of approximately $100 \mathrm{~nm}[1,2]$. On the other hand, the LSPR of particles with various morphologies has been measured with nanometer resolution using electron energy-loss spectroscopy (EELS) with a monochromated electron source in the scanning transmission electron microscope (STEM) [3]. Such a detailed understanding of the effect of the local environment on LSPR is essential in order to optimize particle morphology and particle-support interactions for various applications [4]. We have employed an environmental scanning transmission electron microscope (ESTEM)) to combine high spatial with high energy resolution to measure the effect of support material and gaseous environment on particle LSPR.

Shape-controlled $20 \mathrm{~nm}$ Au NPs were synthesized by reducing Au precursor in the presence of surfactant, Cetyl trimethylammonium bromide and ascorbic acid. Au colloids were mixed with NPs of the support material with different dielectric constants (i.e. $\mathrm{TiO}_{2}, \mathrm{HfO}_{2}$ ) and loaded on lacy carbon TEM grids. All samples were plasma-cleaned to remove the residual surfactant on the Au NP surfaces. In situ STEM-EELS spectra were acquired using an ESTEM equipped with a monochromated, high-brightness electron source (X-FEG) with a $1 \mathrm{~nm}$ beam size and $100 \mathrm{meV}$ energy resolution. LSPR from Au NPs of different shape on different substrates were first measured in vacuum. Then we introduced $\mathrm{H}_{2}$ in the sample area at pressures from $10 \mathrm{~Pa}$ to $100 \mathrm{~Pa}$. Both STEM-EELS spectra and high resolution TEM images were also acquired after the gas was introduced.

Figure 1 is a representative spectrum collected from $\mathrm{Au}$ on $\mathrm{TiO}_{2}$ and $\mathrm{Au}$ on $\mathrm{HfO}_{2}$. It is clear that only one plasmon mode at $2.4 \mathrm{eV}$ (Figure 1a) can be excited for $\mathrm{Au}$ on $\mathrm{TiO}_{2}$ (Figure 1b) while two different modes, $1.5 \mathrm{eV}$ and $2.4 \mathrm{eV}$ (Figure 1a), are excited for similar size and shaped Au NPs supported on $\mathrm{HfO}_{2}$ (Figure 1c). Figure 2a shows the STEM dark-field image of a triangular Au NP on $\mathrm{TiO}_{2}$ support. LSPR measured at two locations, the corner (b) and side (c), of the Au NP were observed to shift to lower energy when the $\mathrm{H}_{2}$ pressure was increased up to 1 mbar (Figure $2 \mathrm{~b}$ and $\mathrm{c}$ ). However, the extent of energy shift at the corner (Figure $2 \mathrm{~b}$ ) was observed to be more than that at the side (Figure 2c) of the NP. Moreover, atomic resolution TEM images show that the surface structure changed after $\mathrm{H}_{2}$ was introduced (Fig 3). Detailed discussion of the LSPR energies for different metal NP-support systems in vacuum and a variety of gases will be presented.

References:

[1]J. A. Scholl et al. Nature 2012, 483, p421

[2] M. Bosman et al. Nanotechnology 2007, 18 
[3] E. Ringe et al. J. Phys. Chem. C 2010, 114, p12511

a)

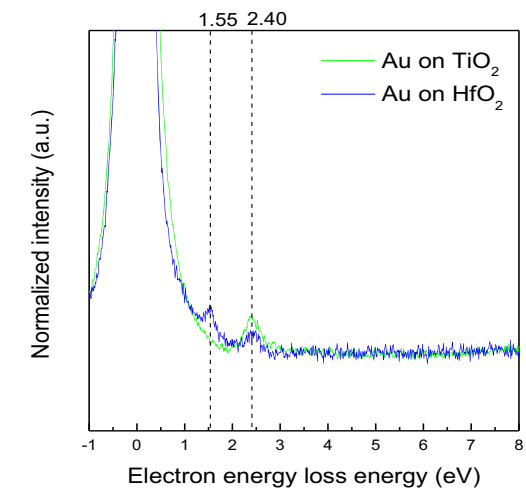

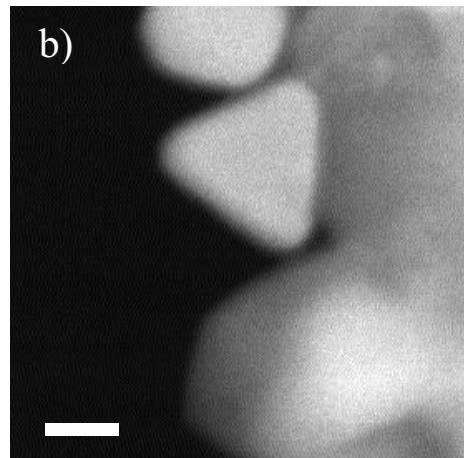

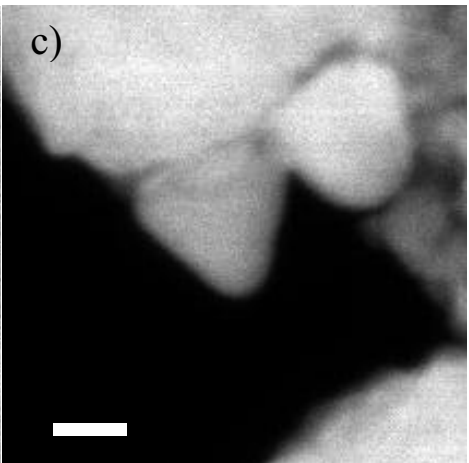

Fig 1. a) STEM-EELS spectra of shaped Au-NP on $\mathrm{TiO}_{2}$ (green) and $\mathrm{HfO}_{2}$ (blue). B) STEM image of $\mathrm{Au}$ on $\mathrm{TiO}_{2}$ c) STEM image of $\mathrm{Au}$ on $\mathrm{HfO}_{2}$. Intensity and position of two spectra are normalized using zero-loss peak. Scale bars are $20 \mathrm{~nm}$.
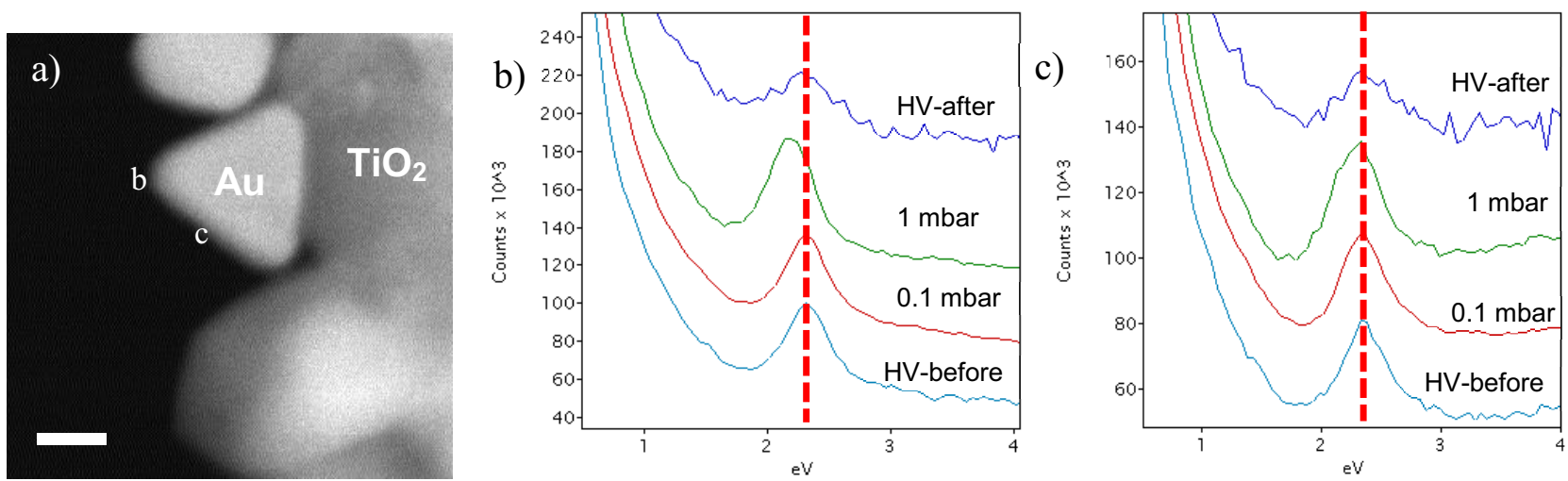

Fig 2. STEM image (a) and EELS spectra (b and c) for $\mathrm{Au}$ on $\mathrm{TiO}_{2}$ substrate in high vacuum (HV), in 0.1 and in $1 \mathrm{mbar}_{2}$, and after $\mathrm{H}_{2}$ pumpint out $\mathrm{H}_{2}$ (HV conditon). a) STEM image, scale bar is 20 $\mathrm{nm}$. b) and c) EELS spectra aquired at location marked ' $b$ ' and ' $c$ ' in the STEM image. The intensities are are shifted arbitrarily and red-dot line aligned at the LSPR energy in HV condition before condition.

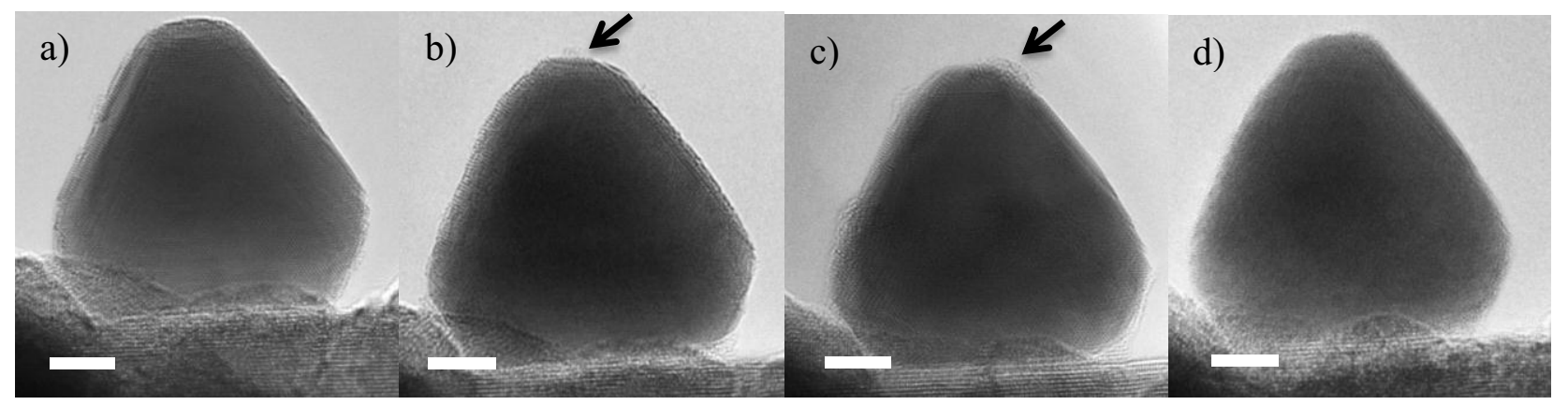

Fig3. High resolution TEM images of a triangular Au NP in (a) HV, before introducing $\mathrm{H}_{2}$ (b) 0.1 mbar $\mathrm{H}_{2}$ (b) 1 mbar $\mathrm{H}_{2}$ (d) $\mathrm{HV}$, after pumping out $\mathrm{H}_{2}$. Note the surface structure change in $\mathrm{H}_{2}$ (marked by arrows). Scale bars are $5 \mathrm{~nm}$. 